\title{
Stage IV T Lymphoblastic Leukemia/Lymphoma
}

National Cancer Institute

\section{Source}

National Cancer Institute. Stage IV T Lymphoblastic Leukemia/Lymphoma. NCI

Thesaurus. Code C8700.

Ann Arbor Classification: Stage IV: Diffuse or disseminated involvement of one or more extralymphatic organs, with or without associated lymph node involvement; or isolated extralymphatic organ involvement in the absence of adjacent regional lymph node involvement, but in conjunction with disease in distant site(s); or any involvement of the liver or bone marrow, or nodular involvement of the lungs(s). - 2003 